\title{
EXPRESSION LEVELS OF ALDOSE REDUCTASE ENZYME, VASCULAR ENDOTHELIAL GROWTH FACTOR AND INTERCELLULAR ADHESION MOLECULE-1 IN THE ANTERIOR LENS CAPSULE OF DIABETIC \\ CATARACT PATIENTS
}

Running title: Cytokines in lens capsule of Diabetic patients

Amr S. Bessa, Msc, PhD (1)

Ahmed M. Ragab, MRCSEd, FRCSEd, Msc $(1,2)$

Rasha A. Nassra (3)

David P. Piñero (4)

Mohamed Shafik Shaheen (1)

\section{Authors' affiliations}

1. Department of Ophthalmology, Faculty of Medicine, Alexandria University, Egypt.

2. Department of ophthalmology, Surrey and Sussex NHS Trust, Associate University Hospital of Brighton and Sussex medical school, United Kingdom.

3. Medical Biochemistry Department, Faculty of Medicine, Alexandria University, Egypt.

4. Department of Optics, Pharmacology and Anatomy, University of Alicante, Spain

Corresponding Author: 
Mohamed Shafik Shaheen, MD, PhD, University of Alexandria, P.O. Box 27, Ibrahimia, Alexandria 21321, Egypt. E-mail: m.shafik@link.net David P. Piñero, PhD Department of Optics, Pharmacology and Anatomy.University of Alicante. Crta San Vicente delRaspeig s/n 03016. San Vicente del Raspeig, Alicante, Spain. Tel: +34-965903500, Fax: +34-965903464 e-mail: david.pinyero@ua.es

Disclosure:

The author David P Piñero has been supported by the Ministry of Economy, Industry and Competitiveness of Spain within the program Ramón y Cajal, RYC2016-20471.

The authors have no proprietary or commercial interest in the medical devices that are involved in this manuscript. 


\section{Abstract}

Purpose: To compare the levels of aldose reductase enzyme (ALR), intercellular adhesion molecule-1 (ICAM-1), and vascular endothelial growth factor (VEGF) in the anterior lens capsule of diabetic versus non-diabetic patients.

Setting: Alexandria main university hospital, Egypt

Design: Prospective case-control study

Methods: This study enrolled 200 eyes of 200 patients undergoing cataract extraction; 51 eyes have proliferative diabetic retinopathy (PDR), 49 eyes have non-proliferative diabetic retinopathy (NPDR), and 100 eyes of non-diabetics. The anterior lens capsules were obtained by performing femtolaser assisted capsulorhexis. Concentrations of ALR, ICAM-1 and VEGF in the lens capsule specimens were measured using human enzyme-linked immunosorbent assay. Results: The mean ALR, ICAM-1 and VEGF levels in the anterior capsule of diabetic group were $2.84 \pm 0.51 \mathrm{ng} / \mathrm{ml}, 87.73 \pm 22.84 \mathrm{pg} / \mathrm{ml}$, and $75.53 \pm 14.95$ $\mathrm{pg} / \mathrm{ml}$, respectively. While in the non-diabetic group they were $1.44 \pm 0.17 \mathrm{ng} / \mathrm{ml}$, $35.45 \pm 2.8 \mathrm{pg} / \mathrm{ml}$, and $33.55 \pm 5.47 \mathrm{pg} / \mathrm{ml}$, respectively. Comparing the concentrations of these mediators, both the PDR and NPDR groups had significantly higher levels compared to the non-diabetic eyes $(p<0.001)$. In addition, eyes with PDR had significantly higher levels of these mediators compared to eyes with NPDR $(p<0.001)$.

Conclusion: The concentrations of ALR, ICAM-1, and VEGF in the anterior lens capsule of diabetic patients are significantly higher than those from nondiabetics. A significantly higher level of three mediators in eyes with PDR 
compared to those with NPDR might allow the use of them as a biomarker for severity of diabetic retinopathy. 


\section{INTRODUCTION}

Diabetes mellitus (DM) is currently a global epidemic which can affect the eye by several mechanisms with cataract and diabetic retinopathy being the most common leading causes of diabetes related visual loss. ${ }^{1}$ Many changes in the retina, retinal vasculature, lens, cornea, and iris have been associated with DM. ${ }^{2}$

Diabetic cataract pathogenesis is believed to be multifactorial though the exact mechanism remains speculative. Many studies suggested a direct link between aldose reductase (ALR) and diabetic cataract development as ALR is considered a key enzyme in diabetic cataract formation and progression. ${ }^{3}$ As a result, the inhibition of ALR has been considered as a potential target for prevention and treatment of diabetes related complications. ${ }^{4}$ ALR has also a detoxification role, as it specifically metabolizes toxic aldehydes, such as hydroxynonenal (HNE), 3-deoxyglucosone, and methylglyosal. However, these aldehydes arise in large quantities from pathological conditions connected with oxidative stress, as in hyperglycemia, and are intermediates for the formation of advanced glycation end products (AGEs). ${ }^{5}$

Vascular endothelial growth factor (VEGF) is a potent vasoactive cytokine that is upregulated under local hypoxic states as in diabetes, causing increased vascular permeability due to endothelial tight junction disruption resulting in extravasation of fluid and retinal edema. ${ }^{6}$ It also stimulates stasis and stickness of blood constitutes in the microvessels of the retina which lead to blood retinal barrier (BRB) breakdown. ${ }^{7}$ Another key inflammatory cytokine that becomes upregulated 
in DM is intercellular adhesion molecule-1 (ICAM-1). ${ }^{8}$ This cytokine has been incriminated in many diabetes mellitus related complications.

The aim of the present study is to compare the levels of ALR, VEGF, and ICAM-1 in the anterior capsule of diabetes mellitus cataract patients compared to non-diabetic senile cataract patients, as well as to assess the correlation of these biomarkers levels with the development and progression of cataract and diabetic retinopathy.

\section{METHODS}

The study was performed at Alexandria main university hospital between November 2015 through April 2017, Alexandria, Egypt. The study adhered to the tenets of the Declaration of Helsinki. Ethical committee approval will be obtained and the patients will be managed according to the ethical guidelines of Alexandria University.

Patients with visually significant cataract undergoing cataract surgery were eligible for enrollment. Visually significant cataract was defined by: any LOCS III grading $\geq 2$ using the slit lamp-based Lens Opacities Classification System III (LOCS III), BCVA <20/40, cataract as the primary cause of vision impairment, and self-reported vision of fair or worse. Both type 2 diabetic patients with varying levels of DR and duration of diabetes between 10 and 15 years but with $\mathrm{HbA} 1 \mathrm{c}$ less than $7.5 \%$ as well as non-diabetic patients were included. Patients undergoing anti-inflammatory treatment and those with systemic disorders including cancer as well as other chronic inflammatory diseases were excluded from the study. Patients who had other ocular 
pathologies, such as pseudoexfoliation syndrome, high myopia > -6.00 diopters, glaucoma, uveitis, trauma, previous ocular surgery, previous intravitreal injection, and any retinopathy other than DR were also excluded from the study. Also, high risk PDR patients and patients with past history or currently have diabetic macular edema were excluded.

Circular anterior capsules, approximately $5.5 \mathrm{~mm}$ in diameter, with attached lens epithelial cells (LECs) were obtained by femtolaser assisted technique after docking (Lensx, Alcon Laboratories, Inc) then the capsules were collected with capsulorhexis forceps (Kawai Capsulorhexis Forceps; Asico, USA). Lens capsule specimens were rinsed with phosphate buffered saline (1X PBS), homogenized at $4{ }^{\circ} \mathrm{C}$ in PBS ( $\left.\mathrm{pH} 7.4\right)$ containing protease inhibitor cocktail to prevent auto-oxidation. Next, the homogenates were centrifuged at $4^{\circ} \mathrm{C}$ for 5 minutes at $5000 \mathrm{xg}$. The supernatant was collected, aliquoted and stored at $80^{\circ} \mathrm{C}$ until biochemical analysis was ready to be performed. Concentrations of ALR, ICAM-1 and VEGF were measured using human enzyme-linked immunosorbent assay (ELISA) kits (CUSABIO, USA, Catalog \#: CSB - E16266h; CUSABIO, USA, Catalog \#: CSB - E04574h and RayBio ${ }^{\circledR}$, Norcross GA, USA, Catalog \#: ELH-VEGF respectively) according to the manufacturer's protocol. The optical density of each sample was determined using a microplate reader at $450 \mathrm{~nm}$ with the correction wavelength set at $540 \mathrm{~nm}$ or $570 \mathrm{~nm}$. The concentration of ALR was calculated based on standards and expressed in $\mathrm{ng} / \mathrm{ml}$, while the concentration of ICAM1 and VEGF were expressed in $\mathrm{pg} / \mathrm{ml}$. The amounts of ALR, ICAM-1 and VEGF in each sample were determined in 
duplicate in each experimental set of plates. The lysate was used for the ELISA assay without prior dilution according to preliminary testing so that sample values fell within the range of the standard curves for each parameter. The ELISA assays used for this study have high sensitivity and excellent specificity for detection of each of the three parameters. No significant cross-reactivity or interference between human ALR, ICAM-1 or VEGF and analogues or other cytokines was observed.

Statistical analysis: The Statistical Package for Social Sciences for Windows (SPSS Inc., PASW Statistics, version 18) was used for statistical analysis, and findings were considered to be statistically significant if $P<0.05$. The differences in the expression of ALR, ICAM-1, and VEGF between the DM and control groups were analyzed by Student's $t$-test, Mann-Whitney U-test, and Pearson's Chi-square test, as appropriate. The correlations among ALR, ICAM-1, and VEGF expression were analyzed by Spearman's rank correlation test. The receiver operator characteristic (ROC) curve was used to determine the optimal cut-off value of ALR, ICAM-1and VEGF expressed in the LECs, according to the coordinates of the cut-off point of the ROC curve that was closest to the coordinate $(0,1)$. The odds ratios of the expression of ALR, ICAM-1 and VEGF in the LECs were also evaluated as risk factors associated with the progression of cataract in type 2 diabetes between the DM and the control groups as well as between proliferative and non-proliferative diabetic retinopathy subgroups.

\section{Results}


A total of 200 patients were eligible for inclusion in this study. They were divided into two main groups. The first group included 100 eyes of 100 patients who were diabetic for ten years and had visually significant cataracts. This group was further subdivided into two subgroups: one included 49 eyes diagnosed with non-high-risk PDR and the other included 51 eyes diagnosed with NPDR. The second main group included 100 eyes of 100 non-diabetic patients with visually significant cataracts (control group). Patients in group 1 included 44 women and 46 men with mean age $50 \pm 7.3$ years. Group 2 (control group) included 50 women and 50 men with an average age of $53.4 \pm 5.2$ years. There were no significant differences between the groups as regard age and gender.

The mean ICAM-1 level from the anterior capsule was $87.73 \pm 22.84 \mathrm{pg} / \mathrm{ml}$ in the diabetic group and $35.45 \pm 2.81 \mathrm{pg} / \mathrm{ml}$ in the control group $(p<0.001)$. The mean ALR level was $2.84 \pm 0.51 \mathrm{ng} / \mathrm{ml}$ in diabetic group and $1.44 \pm 0.17 \mathrm{ng} / \mathrm{ml}$ in the control group $(p<0.001)$. The mean VEGF level was $75.53 \pm 14.95 \mathrm{pg} / \mathrm{ml}$ in diabetic group and $33.55 \pm 5.47 \mathrm{pg} / \mathrm{ml}$ in the control group $(p<0.001)$. The difference in levels remained significant when comparing the control group to eyes with either PDR or NPDR separately (Tables 1 and 2)

ROC curve analysis and odds ratio of ICAM-1, ALR, and VEGF expression in LECs: Following analysis of the ROC curve, the optimal cut-off values of ICAM-1, ALR, and VEGF expression in LECs needed to differentiate between diabetic and non-diabetic cataract groups were found to be $>42.5 \mathrm{pg} / \mathrm{ml},>1.8$ $\mathrm{ng} / \mathrm{ml}$, and $>32.5 \mathrm{pg} / \mathrm{ml}$ respectively. 
The AUC for ICAM-1 was 0.985 ( $p<0.001)$ with a sensitivity of $96 \%$, specificity of 97\%, positive predictive value (PPV) of $97 \%$, and negative predictive value (NPV) of $96 \%$. The AUC for ALR was $0.988(p<0.001)$ with a sensitivity of $92 \%$, specificity of $100 \%$, PPV of $100 \%$, and NPV of $92.6 \%$. The AUC for VEGF was $0.983(p<0.001)$ with a sensitivity of $97 \%$, specificity of $66 \%$, PPV of $74 \%$, and NPV of $95.7 \%$ (Figure 1).

When the ROC curve was analyzed to predict the cut off values of ICAM-1, ALR and VEGF expression in LECs comparing PDR and NPDR subgroups, the values were $>90 \mathrm{pg} / \mathrm{ml},>2.8 \mathrm{ng} / \mathrm{ml}$ and $>72.5 \mathrm{pg} / \mathrm{ml}$ respectively. ICAM-1 had the highest sensitivity and specificity as a predictor of proliferative changes in diabetic cataract (98\% and 100\% respectively) (Figures 2 and 3).

Moreover, the odds ratios for the expression of ALR and VEGF in the LECs were 11.086 -fold and 27.547-fold higher in the PDR group, respectively, than in the NPDR group. Thus, these mediators may be regarded as important risk factors during the progression of cataract in diabetic patients (Table 3)

\section{Discussion}

The development of DR is a complex process involving many mediators including different growth factors, genetic predisposition, and immune response. ${ }^{9}$ In order to elucidate the relation between the immune cells, vascular endothelial cells, and the role of ALR enzyme in the lens capsule of DR patients, this study investigated the expression of ALR, VEGF and ICAM-1 in anterior lens capsules from diabetic versus non-diabetic patients undergoing cataract surgery. The 
study found that the levels of ALR, VEGF, and ICAM-1 were significantly higher in the diabetic group compared to the non-diabetic control group. Moreover, the level of these mediators was also significantly higher in the anterior lens capsule of eyes with PDR compared to those with NPDR.

The elevated levels of ALR seen with worsening levels of DR correlate with the common understanding of its role in diabetic cataract formation. ALR is a rate-limiting enzyme of the polyol pathway. ALR-mediated polyol accumulation has been shown to lead to sorbitol accumulation in diabetic eyes causing increased osmotic pressure, alterations in cell membrane permeability, edema, and damage to cells of the crystalline lens. ${ }^{10}$ Intracellular accumulation of sorbitol primarily occurs under hyperglycemic conditions which in turn leads to acceleration of cataract formation and progression. ${ }^{11}$ Therefore, the increased ALR levels may correlate with the presumed higher levels of chronic hyperglycemia that are likely found in PDR compared to NPDR and non-diabetic controls.

Correlation between increasing levels of ALR with the development and progression of DR is still disputable. Previous studies which tried to investigate the associations between ALR gene polymorphism and DR ended up with controversial results. One study recruited 3512 DM patients with DR and 4319 DM patients without DR. The study found that $A L R C(-106)$ T polymorphism was not associated with an increased risk of DR. However, subgroup analysis demonstrated a genetic association. ${ }^{12}$ Another study conducted by Abhary et al 
found that variations within the $A K R 1 B 1$ gene were highly associated with diabetic retinopathy development irrespective of ethnicity. ${ }^{13}$

Increased levels of VEGF with worsening DR seen in the current study may also correlate with the hyperglycemic environment. Hyperglycemia induced oxidative stress leads to formation of advanced glycation end products (AGEs). ${ }^{7}$ Recent studies suggest that AGEs interact with plasma membrane localized receptors for AGEs (RAGE) to alter intracellular signaling, gene expression, release of pro-inflammatory molecules and free radicals. As a result, AGEs may alter cellular functions in LECs under local hypoxic conditions as seen in diabetics, leading to increased levels of inflammatory cytokines, AGEs and reactive oxygen species. Consequently, this may induce VEGF gene expression which promotes angiogenesis and vascular permeability. ${ }^{6,14}$ That is why VEGF nowadays is an attractive target for many studies aiming at halting DM related ocular complications. ${ }^{15}$

The higher levels of ICAM-1 with worsening DR as seen in this study also correlates with the fact that its upregulation in inflammatory states. One of the key inflammatory events involved in the BRB alteration appears to be increased expression of endothelial adhesion molecules including ICAM-1 in response to some of these cytokines. ${ }^{8}$ ICAM-1 promotes retinal vascular leukocyte adhesion, increased vascular permeability, and capillary closure in combination with elevated VEGF levels. ${ }^{16,17}$ In fact, VEGF may potentiate the expression of ICAM1 in the retinal vasculature. ${ }^{18}$ Increased levels of soluble ICAM-1 in the serum of diabetic patients with severe complications have been documented. ${ }^{19}$ In addition, 
it is elevated in both the aqueous humor and vitreous of diabetic patients. ${ }^{20,21}$ More recently, a study by Hiller et al correlated increased aqueous humor ICAM1 with severity of diabetic macular edema. ${ }^{22}$

The current study has several limitations. While ALR, VEGF, and ICAM-1 are significantly elevated in the anterior lens capsules of eyes with DR, this study was not designed to determine if these mediators are directly involved in the pathogenesis of diabetic cataracts or DR. It is possible that these mediators may merely reflect the severity of hyperglycemia, inflammation and ischemia in eyes with DR. The duration of diabetes and standardized scoring of DR severity as indicators of glycemic control were also not addressed in this study, which may have affected the expression of the studied mediators. In any case, it should be considered that only patients with $\mathrm{HbA} 1 \mathrm{c}$ less than $7.5 \mathrm{mg} / \mathrm{dl}$ were enrolled in the study to ensure a good glycemic control. This can reflect a good control of diabetes three months preceding the surgery. Another potential limitation was the fact that a "visually significant" cataract was determined by the surgeon and not based on standard criteria. It is possible that variations in the severity of the cataract in the different studied groups could have also affected the results. In conclusion, the levels of ALR, VEGF and ICAM-1 in the anterior lens capsule are significantly higher in diabetic compared with non-diabetic eyes. The fact that the levels of all three were significantly higher in PDR eyes compared to NPDR eyes suggests that these mediators may serve as important biomarkers for severity of diabetic retinopathy. Future studies will be necessary to determine whether the 
elevated levels of these mediators are directly responsible for diabetic cataract formation and worsening DR or a reaction to the intraocular milieu.

\section{WHAT WAS KNOWN}

-A direct link seems to be present between aldose reductase (ALR) and diabetic cataract development as ALR is considered a key enzyme in diabetic cataract formation and progression.

-Vascular endothelial growth factor (VEGF) is upregulated under local hypoxic states as in diabetes

-Intercellular adhesion molecule-1 (ICAM-1) is another key inflammatory cytokine that becomes upregulated in diabetes mellitus.

\section{WHAT THIS PAPER ADDS}

-Levels of ALR, VEGF and ICAM-1 in anterior lens capsules are significantly higher in eyes from diabetic patients compared to the non-diabetic. Moreover, the level of these mediators was also significantly higher in the anterior lens capsule of eyes with proliferative diabetic retinopathy (PDR) compared to those with non-proliferative diabetic retinopathy (NPDR).

-The study of these inflammatory mediators may help for a better understanding of diabetes-related eye complications. In other words, the 
presence of these mediators in significant amount in diabetic eyes might indicate an association between them and the pathogenesis of diabetic eye disease.

-The levels of these three mediators may serve as important biomarkers for severity of diabetic retinopathy. 


\section{REFERENCES}

1. Vieira-Potter VJ, Karamichos D, Lee DJ. Ocular complications of diabetes and therapeutic approaches. Biomed Res Int 2016;2016:3801570.

2. Pollreisz A, Schmidt-Erfurth U. Diabetic cataract-pathogenesis, epidemiology and treatment. J Ophthalmol 2010;2010:608:751.

3. Reddy AB, Tammali R, Mishra R, Srivastava S, Srivastava SK, Ramana KV. Aldose reductase deficiency protects sugar-induced lens opacification in rats. Chem Biol Interact 2011;191:346-50.

4. Kawakubo K, Mori A, Sakamoto K, Nakahara T, Ishii K. GP-1447, an inhibitor of aldose reductase, prevents the progression of diabetic cataract in rats. Biol Pharm Bull 2012;35:866:72.

5. Hotta N, Akanuma Y, Kawamori R, Matsuoka K, Oka Y, Shichiri M, Toyota T, Nakashima M, Yoshimura I, Sakamoto N, Shigeta Y. Long-termclinical effects of epalrestat, an aldose reductase inhibitor, on diabetic peripheral neuropathy: the 3year, multicenter, comparative aldose reductase inhibitor-diabetes complications trial. Diabetes Care 2006;29:1538-44.

6. Caldwell RB, Bartoli M, Behzadian MA, El-Remessy AE, Al-Shabrawey M, Platt $\mathrm{DH}$, Caldwell RW. Vascular endothelial growth factor and diabetic retinopathy: pathophysiological mechanisms and treatment perspectives. Diabetes Metab Res Rev 2003;19:442-55. 
7. Yan SD, Schmidt AM, Anderson GM, Zhang J, Brett J, Zou YS, Pinsky D, Stern

D. Enhanced cellular oxidant stress by the interaction of advanced glycation end products with their receptors/binding proteins. J Biol Chem 1994;269:9889-97.

8. Zhang Q, Ames JM, Smith RD, Baynes JW, Metz TO. A perspective on the Maillard reaction and the analysis of protein glycation by mass spectrometry: probing the pathogenesis of chronic disease. J Proteome Res 2009;8:754-69.

9. From the Centers for Disease Control and Prevention: Blindness caused by diabetes-- Massachusetts, 1987-1994. JAMA 1996;276:1865-6.

10. Anil Kumar P, Bhanuprakash Reddy G. Focus on molecules: aldose reductase. Exp Eye Res 2007;85:739-40.

11. Kador PF, Kinoshita JH. Role of aldose reductase in the development of diabetes-associated complications. Am J Med 1985;79:8-12.

12. Zhou M, Zhang P, Xu X, Sun X. The Relationship Between Aldose

Reductase C106T Polymorphism and Diabetic Retinopathy: An Updated MetaAnalysis. Invest Ophthalmol Vis Sci 2015;56:2279-89.

13. Abhary S, Hewitt AW, Burdon KP, Craig JE. A systematic meta-analysis of genetic association studies for diabetic retinopathy. Diabetes 2009;58:2137-47. 14. Aiello LP, Avery RL, Arrigg PG, Keyt BA, Jampel HD, Shah ST, Pasquale LR, Thieme $\mathrm{H}$, Iwamoto MA, Park JE, et al. Vascular endothelial growth factor in ocular fluid of patients with diabetic retinopathy and other retinal disorders. $\mathrm{N}$ Engl J Med 1994;331:1480-87.

15. Pober JS, Sessa WC. Evolving functions of endothelial cells in inflammation. Nat Rev Immunol 2007;7:803-15. 
16. Joussen AM, Poulaki V, Qin W, Kirchhof B, Mitsiades N, Wiegand SJ, Rudge J, Yancopoulos GD, Adamis AP. Retinal vascular endothelial growth factor induces intercellular adhesion Molecule-1 and endothelial nitric oxide synthase expression and initiates early diabetic retinal leukocyte adhesion in vivo. Am J Pathol 2002;160:501-9.

17. Miyamoto K, Khosrof S, Bursell SE, Moromizato Y, Aiello LP, Ogura Y, Adamis AP. Vascular endothelial growth factor (VEGF)-induced retinal vascular permeability is mediated by intercellular adhesion molecule-1 (ICAM-1). Am J Pathol 2000;156:1733-9.

18. Lu M, Perez VL, Ma N, Miyamoto K, Peng HB, Liao JK, Adamis AP. VEGF increases retinal vascular ICAM-1 expression in vivo. Invest Ophthalmol Vis Sci 1999;40:1808-12.

19. Gearing AJH, Hemmingway I, Pigott R, Hughes J, Rees AJ, Cashman SJ. Soluble forms of vascular adhesion molecules, E-Selectin, ICAM-1 and VCAM-1: pathological significance. Annals New York Acad 1992;667:324-31.

20. Jonas JB, Jonas RA, Neumaier M, Findeisen P. Cytokine concentration in aqueous humor of eyes with diabetic macular edema. Retina 2012;32:2150-7. 21. Funatsu $\mathrm{H}$, Noma $\mathrm{H}$, Mimura $\mathrm{T}$, Eguchi $\mathrm{S}$, Hori $\mathrm{S}$. Association of vitreous inflammatory factors with diabetic macular edema. Ophthalmology 2009;116:739.

22. Hillier RJ, Ojaimi E, Wong DT, Mak MY, Berger AR, Kohly RP, Kertes PJ, Forooghian F, Boyd SR, Eng K, Altomare F, Giavedoni LR, Nisenbaum R, Muni 
$\mathrm{RH}$. Aqueous humor cytokine levels as biomarkers of disease severity in diabetic macular edema. Retina 2017;37:761-9. 
Figure legends:

Figure (1): $\quad$ ROC curve for ICAM - 1, ALR and VEGF to predict diabetes

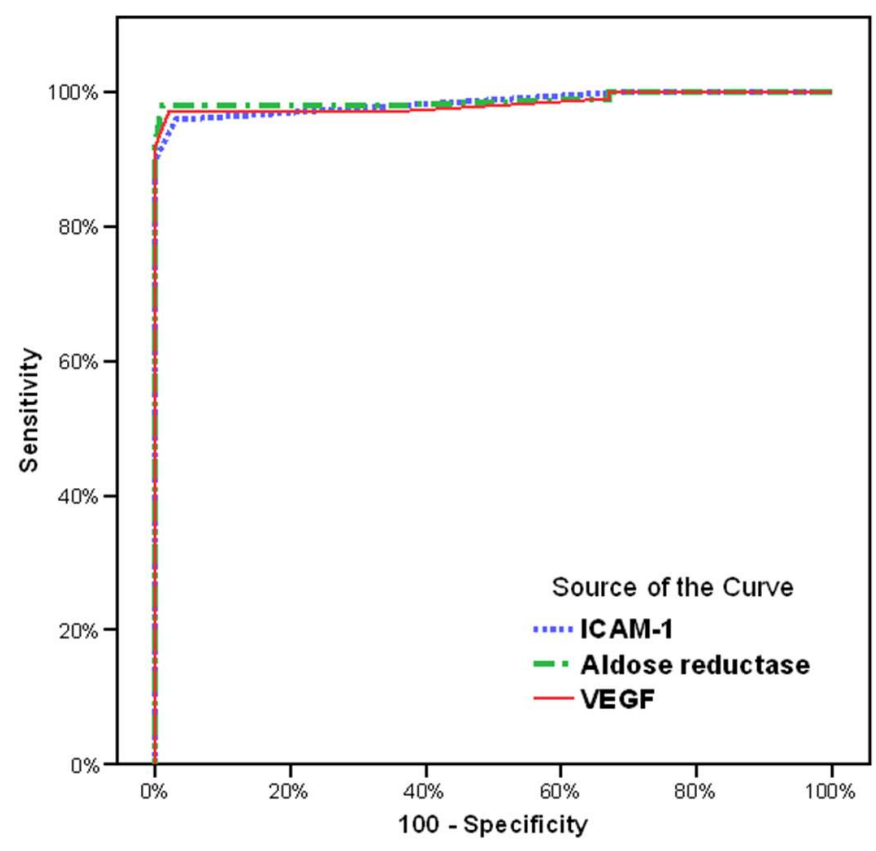

Figure (2): $\quad$ ROC curve for ICAM - 1, ALR and VEGF to predict PDR

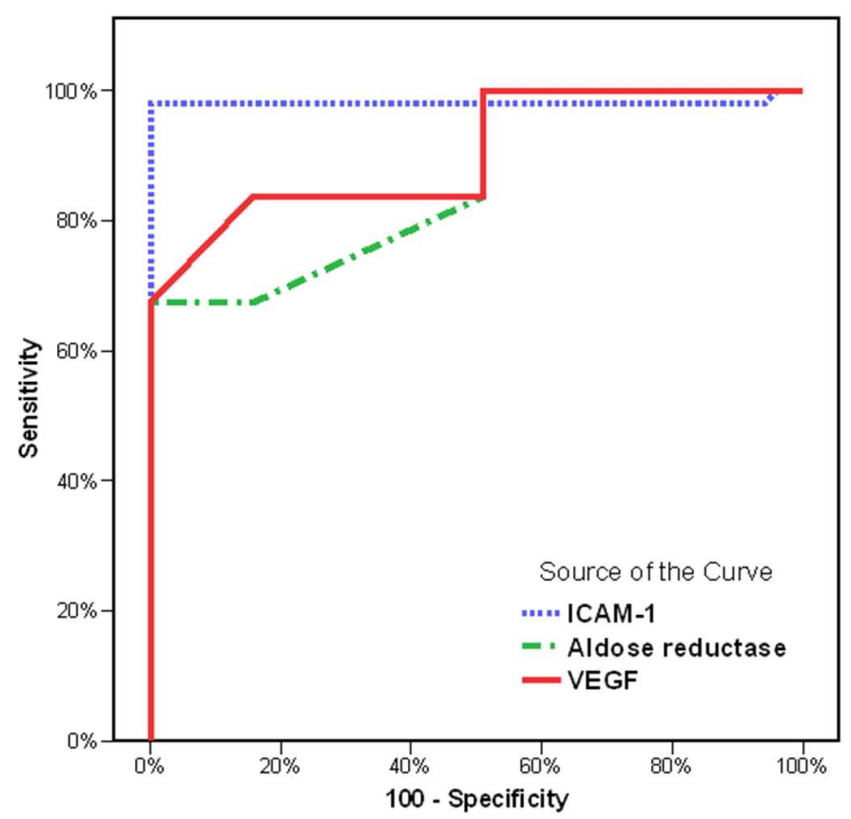


Figure (3): Comparison between the studied subgroups according to the different parameters

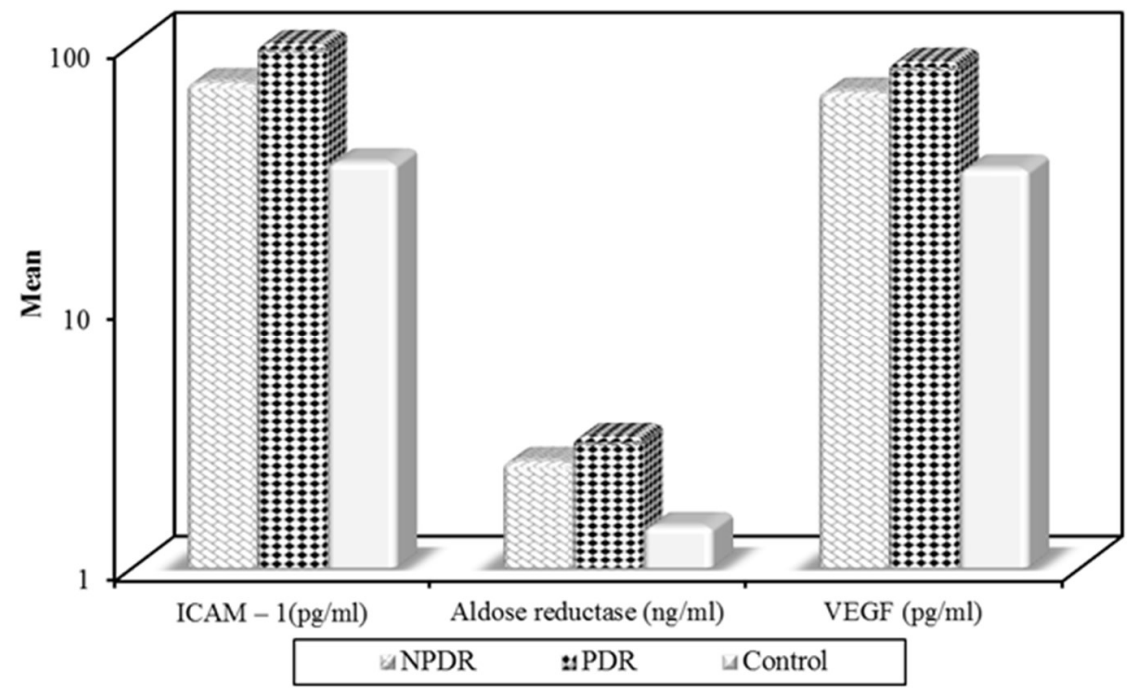


Table (1): Comparison of ICAM-1, aldose reductase, and VEGF in diabetics versus non-diabetics.

\begin{tabular}{llll}
\hline & $\begin{array}{l}\text { Diabetes } \\
(\mathrm{n}=100)\end{array}$ & $\begin{array}{l}\text { Control } \\
(\mathrm{n}=100)\end{array}$ & $\mathrm{P}$ \\
\hline ICAM $-1(\mathrm{pg} / \mathrm{ml})$ & $87.73 \pm 22.84$ & $35.45 \pm 2.81$ & $<0.001^{*}$ \\
$\begin{array}{l}\text { Aldose reductase } \\
(\mathrm{ng} / \mathrm{ml})\end{array}$ & $2.84 \pm 0.51$ & $1.44 \pm 0.17$ & $<0.001^{*}$ \\
VEGF $(\mathrm{pg} / \mathrm{ml})$ & $75.53 \pm 14.95$ & $33.55 \pm 5.47$ & $<0.001^{*}$ \\
\hline
\end{tabular}

Normally quantitative data was expressed in mean \pm SD and was compared using student t-test. *: Statistically significant at $p \leq 0.05$. ICAM-1: intercellular adhesion molecule-1. VEGF: vascular endothelial growth factor. pg: picogram. ng: Nanogram.

Table 2: Comparison of ICAM-1, aldose reductase, and VEGF in non-proliferative diabetic retinopathy versus proliferative diabetic retinopathy versus non-diabetic controls.

\begin{tabular}{|c|c|c|c|c|}
\hline & $\begin{array}{l}\text { NPDR } \\
(n=51)\end{array}$ & $\begin{array}{c}\text { PDR } \\
(n=49)\end{array}$ & $\begin{array}{l}\text { Control } \\
(n=100)\end{array}$ & $\mathbf{p}$ \\
\hline ICAM - 1(pg/ml) & $71.57 \pm 16.74$ & $104.54 \pm 14.75$ & $35.45 \pm 2.81$ & $<0.001^{*}$ \\
\hline Sig. between groups & \multicolumn{3}{|c|}{$\mathrm{p}_{1}<0.001^{*}, \mathrm{p}_{2}<0.001^{*}, \mathrm{p}_{3}<0.001^{*}$} & \\
\hline Aldose reductase (ng/ml) & $2.55 \pm 0.41$ & $3.15 \pm 0.43$ & $1.44 \pm 0.17$ & $<0.001^{*}$ \\
\hline Sig. between groups & \multicolumn{3}{|c|}{$\mathrm{p}_{1}<0.001^{*}, \mathrm{p}_{2}<0.001^{*}, \mathrm{p}_{3}<0.001^{*}$} & \\
\hline VEGF(pg/ml) & $65.98 \pm 11.57$ & $85.46 \pm 11.13$ & $33.55 \pm 5.47$ & $<0.001^{*}$ \\
\hline Sig. between groups & \multicolumn{3}{|c|}{$\mathrm{p}_{1}<0.001^{*}, \mathrm{p}_{2}<0.001^{*}, \mathrm{p}_{3}<0.001^{*}$} & \\
\hline
\end{tabular}

Normally quantitative data was expressed in mean $\pm \mathrm{SD}$ and was compared using $\mathrm{F}$ value for ANOVA test, Post Hoc Test (LSD).

$p_{1}: p$ value for comparing between NPDR and PDR

$\mathrm{p}_{2}: \mathrm{p}$ value for comparing between NPDR and Control

$\mathrm{p}_{3}$ : $\mathrm{p}$ value for comparing between PDR and Control

*: Statistically significant at $\mathrm{p} \leq 0.05$ 
Table (3): $\quad$ Odds Ratio (OR) analysis between NPDR and PDR subgroups

\begin{tabular}{|c|c|c|c|c|c|c|c|}
\hline & \multicolumn{2}{|c|}{$\begin{array}{l}\text { NPDR } \\
(n=51)\end{array}$} & \multicolumn{2}{|c|}{$\begin{array}{l}\text { PDR } \\
(n=49)\end{array}$} & \multirow[t]{2}{*}{$P$} & \multirow[t]{2}{*}{ OR } & \multirow{2}{*}{$\begin{array}{l}95 \% \mathrm{Cl} \\
\text { (LL-UL) }\end{array}$} \\
\hline & No & $\%$ & No & $\%$ & & & \\
\hline \multicolumn{8}{|l|}{ ICAM } \\
\hline$\leq 90$ & 51 & 100 & 1 & 2.0 & \multirow{3}{*}{$<0.001^{*}$} & & \\
\hline$>90$ & 0 & 0.0 & 48 & 98.0 & & - & - \\
\hline \multicolumn{7}{|l|}{ Aldose } & \\
\hline$\leq 2.8$ & 43 & 84.3 & 16 & 32.7 & \multirow{2}{*}{$0.001^{*}$} & & \\
\hline$>2.8$ & 8 & 15.7 & 33 & 67.3 & & 11.086 & $4.2-29.0$ \\
\hline \multicolumn{8}{|l|}{ VEGF } \\
\hline$\leq 72.5$ & 43 & 84.3 & 8 & 16.3 & \multirow{2}{*}{$0.001^{*}$} & & \\
\hline$>72.5$ & 8 & 15.7 & 41 & 83.7 & & 27.547 & $9.4-80.3$ \\
\hline
\end{tabular}

